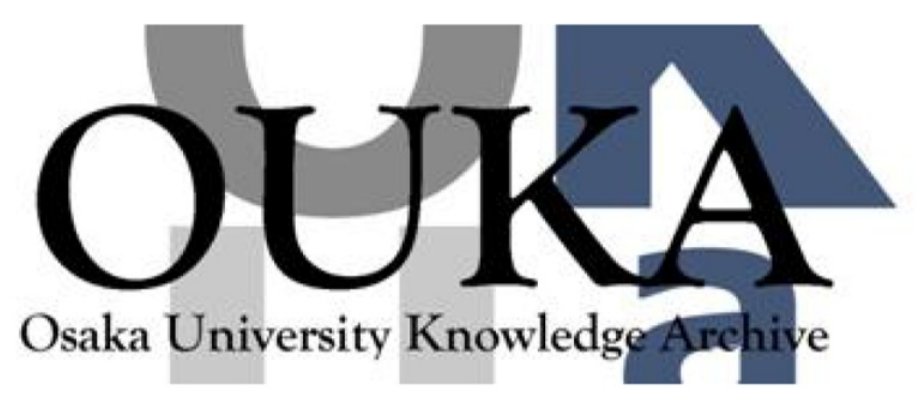

\begin{tabular}{|c|l|}
\hline Title & $\begin{array}{l}\text { Measurements of laser-hole boring into } \\
\text { overdense plasmas using x-ray laser } \\
\text { refractometry(invited) }\end{array}$ \\
\hline Author(s) & Kodama, R.; Takahashi, K.; Tanaka, K. A. et al. \\
\hline Citation & $\begin{array}{l}\text { Review of Scientific Instruments. 70(1) p. 543- } \\
\text { p.548 }\end{array}$ \\
\hline Issue Date & $1999-01$ \\
\hline oaire:version & VoR \\
\hline URL & https://hdl. handle.net/11094/3462 \\
\hline rights & \\
\hline Note & \\
\hline
\end{tabular}

Osaka University Knowledge Archive : OUKA

https://ir. Library. osaka-u. ac. jp/

Osaka University 


\title{
Measurements of laser-hole boring into overdense plasmas using $x$-ray laser refractometry (invited)
}

\author{
R. Kodama, K. Takahashi, K. A. Tanaka, and Y. Kato \\ Institute of Laser Engineering (ILE), Osaka University, Suita, Osaka 565, Japan \\ K. Murai \\ DMP, ONRI, Ikeda, Osaka 563, Japan \\ F. Weber, T. W. Barbee, and L. B. DaSilva \\ Lawrence Livermore National Laboratory, University of California, Livermore, California 94550
}

(Presented on 9 June 1998)

\begin{abstract}
We developed a $19.6 \mathrm{~nm}$ laser $\mathrm{x}$-ray laser grid-image refractometer (XRL-GIR) to diagnose laser-hole boring into overdense plasmas. The XRL-GIR was optimized to measure two-dimensional electron density perturbation on a scale of a few tens of $\mu \mathrm{m}$ in underdense plasmas. Electron density profiles of laser-produced plasmas were obtained for $10^{20}-10^{22} \mathrm{~cm}^{-3}$ with the XRL-GIR and for $10^{19}-10^{20} \mathrm{~cm}^{-3}$ from an ultraviolet interferometer, the profiles of which were compared with those from hydrodynamic simulation. By using this XRL-GIR, we directly observed laser channeling into overdense plasmas accompanied by a bow shock wave showing a Mach cone ascribed to supersonic propagation of the channel front. (c) 1999 American Institute of Physics. [S0034-6748(99)61901-3]
\end{abstract}

\section{INTRODUCTION}

Investigation of electron density profiles as basic parameters is quite important to the study of physical processes in laser-plasma interactions. Optical probe systems such as shadowgraphy and interferometry have been developed and are used to measure the density profiles in underdense plasmas, which was useful for the laser-plasma interaction experiments in previous work. ${ }^{1}$ Laser-channel formation in underdense plasmas, for example, has been experimentally studied well by using these optical probe systems. ${ }^{2}$ However, recent progress in short pulse intense-laser technologies has increased the electron density of the interaction plasmas. At laser intensities above $I_{L} \lambda^{2}>10^{17} \mathrm{~W} \mu \mathrm{m}^{2} / \mathrm{cm}^{2}$, where $I_{L}$ is the laser intensity and $\lambda$ the wavelength of the laser light, electron quiver motions dominate thermal motions and enormous photon pressure will push the turning point forward into the overdense region (laser-hole boring) as well as evacuate the plasma radially. ${ }^{3}$ Ignoring the thermal pressure of the plasma compared with the photon pressure, the propagation of the laser-turning point is given simply by a momentum conservation (snow plow model) as

$$
\frac{d}{d t}\left(\int_{0}^{x(t)} \frac{x}{L} \rho_{c} d x[2 x(\dot{t})]\right)=P_{L}=(1+\xi) \frac{I_{L}}{c},
$$

where $L$ is the linear scale length of the plasma, $\rho_{C}$ the critical mass density, and $\xi$ the reflectivity of the laser light at the turning point. This simple model indicates that $1 \mu \mathrm{m}$ laser light with a pulse duration of $100 \mathrm{ps}$ at an intensity of $10^{18} \mathrm{~W} / \mathrm{cm}^{2}$ can propagate and create a channel in a $100 \mu \mathrm{m}$ scale-length plasma for a distance of $100 \mu \mathrm{m}$ into the overdense plasmas. Then, the intense-laser light in the channel interacts with plasmas surrounded by overcritical density plasmas. This increase in the density of the surrounding plasma will make it more difficult to investigate the highintensity laser-plasma interactions as compared with conventional plasma interactions. Previous techniques such as optical interferometry can measure the plasma profiles only at electron densities $<10^{21} \mathrm{~cm}^{-3}$ taking into account the significant refraction of the probe light in the gradient largescale plasma even though the critical density for the probe light is much higher than the detectable density.

Fortunately, further progress in laser technology, i.e., use of collisionally pumped extreme ultraviolet (XUV) laser light, is opening new possibilities of actively diagnosing such overdense plasmas. The laser systems have been demonstrated to operate at wavelengths ranging from 3.5 to 40 $\mathrm{nm} .{ }^{4} \mathrm{X}$-ray laser light has the advantage of brightness and small beam divergence as a light source in the probe system for measurement of the high density plasma as well as short wavelength. Hence, new probe systems using x-ray laser light have been developed to measure one-dimensional density profiles with moiré deflectometry, ${ }^{5}$ two-dimensional area density distribution in a thin plasma with shadowgraphy, ${ }^{6}$ and two-dimensional density profiles with $\mathrm{x}$-ray interferometry. ${ }^{7}$ Deflectometry and interferometry, which are sensitive to the refractive index or to gradients in the refractive index, will be more appropriate for obtaining quantitative information on density gradients than shadowgraphy. Interferometry using $\mathrm{x}$-ray laser light must be most suitable for measurements of two-dimensional density profiles in an overdense region, but it is much more demanding on the experimental arrangement and x-ray optics than deflectometry. A moiré deflectometry system using $\mathrm{x}$-ray laser light has a more simple arrangement than interferometry. However, this technique (moiré) is limited for measurements of density perturbation since it produces a signal proportional to the electron density gradient along only one dimension. So 
we have developed an $\mathrm{x}$-ray laser light grid-image refractometry (XRL-GIR) to obtain two-dimensional spatial information on the density gradient to investigate channel formation in the overdense region.

In Sec. II, we discuss the main advantages of x-ray laser refractometry compared to shadowgraphy using an x-ray laser illumination source to measure the laser channel in the long-scale overdense plasma. In Sec. III we present the concept behind the XRL-GIR system to be optimized for the channeling experiments. In Secs. IV and V, the setup for the interaction experiments and the results of the laser channel tests into the overdense region are shown, respectively. A summary and expectations for future developments are presented in Sec. VI.

\section{X-RAY REFRACTOMETRY FOR OVERDENSE PLASMAS}

At laser intensities of $>10^{17-18} \mathrm{~W} / \mathrm{cm}^{2}, 1 \mu \mathrm{m}$ laser light can create a hole in long scale-length plasmas at densities of $10^{21}-10^{22} \mathrm{~cm}^{-3}$ as indicated by Eq. (1). These plasmas with a few $100 \mu \mathrm{m}$ scale will exhibit both strong absorption and significant refraction at conventional probe wavelengths $(0.25-1 \mu \mathrm{m})$. Both absorption and the refraction of optical beams are reduced by using shorter probe beam wavelengths, which is the principal advantage of the use of soft $\mathrm{x}$-ray (XUV) sources for probing these plasmas. However, absorption and refraction must be considered even at the shorter XUV wavelengths since they ultimately limit the size or density of the plasma or plasma density perturbation that can be probed. Absorption places limits primarily on the maximum density of the plasma while refraction places limits on the magnitude of the density gradients that can be tolerated. The sizes and the densities of the modulated and nonmodulated plasmas, then, decide the more appropriate technique, shadowgraphy (absorption) or refractometry (refraction) using XUV wavelengths, to show plasma perturbation such as laser-hole boring.

One can simply estimate the absorption of the probe light in the plasma with only bremsstrahlung opacities ${ }^{8}$ eliminating any bound-free absorption in high temperature plasmas. Assuming a $\mathrm{CH}$ cylindrical plasma at $100 \mathrm{eV}$ with a scale of $100 \mu \mathrm{m}$, a diameter of $400 \mu \mathrm{m}$, and no refraction effect on the probe beam, only $2 \%$ of the intensity of the original laser light at a wavelength of $20 \mathrm{~nm}$ can be transmitted through this plasma. This significant absorption will limit both shadowgraphy and refractometry from the point of view of the detection limit of the probe signal compared with the level of the noise due to self-emission from the probed plasmas. We compare both shadowgraphy and refractometry techniques using a $20 \mathrm{~nm}$ probe light for the $\mathrm{CH}$ plasmas (400 $\mu \mathrm{m}$ diameter $/ 100 \mathrm{eV}$ ) with a vacuum hole at the center of the plasma column corresponding to laser channeling. An exponential profile with a scale length of $100 \mu \mathrm{m}$ is assumed as the density gradient of the plasma column in order to estimate the refraction. The width of the laser-channel hole is varied from 0 to $150 \mu \mathrm{m}$ at the center of the plasma column. Figure 1 shows a comparison of x-ray laser refractometry and shadowgraphy for the laser channel measurements from

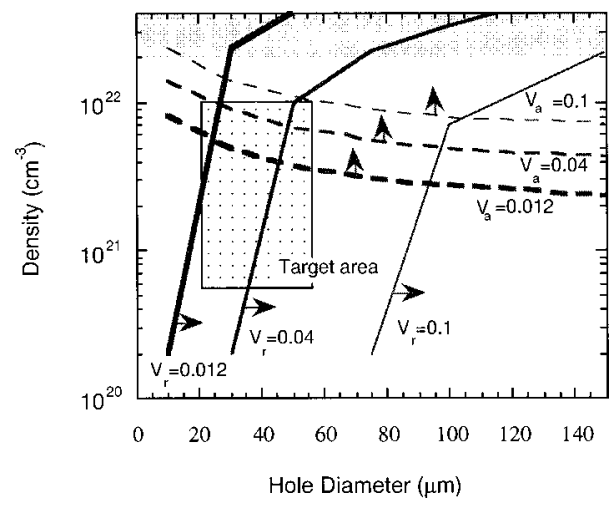

FIG. 1. Comparison of refraction and absorption methods for a $20 \mathrm{~nm}$ laser beam to probe a laser channel in $100 \mathrm{eV} \mathrm{CH}$ plasmas. A cylindrical plasma with a diameter of $400 \mu \mathrm{m}$ and a scale length of $100 \mu \mathrm{m}$ is assumed. The hole diameter is changed from 0 to $150 \mu \mathrm{m}$ at the center of the plasma column.

ray-trace calculations. The calculation for shadowgraphy is the ratio of the transmission of the probe beam changing electron densities as a function of the hole diameter given by

$$
\frac{\left|I_{h}-I_{n}\right|}{I_{h}+I_{n}}=v_{a},
$$

where $I_{n}$ is the intensity of the probe light through the plasma column beside the hole and $I_{h}$ is the intensity transmitted though the plasma with a vacuum hole. The dotted lines in Fig. 1 correspond to $v_{a}=0.1,0.04$, and 0.012 , respectively. The absorption method becomes more effective with a higher density region. At hole widths of more than $100 \mu \mathrm{m}$, this method depends only on the density. Another estimation for refractometry from ray tracing is the ratio of the deflection angle of the probe beam given by

$$
\frac{\left|\Delta \phi_{n}-\Delta \phi_{h}\right|}{\Delta \phi_{h}+\Delta \phi_{n}}=v_{r}
$$

where $\Delta \phi_{n}$ is the deflection angle of the probe beam through the plasma beside the hole and $\Delta \phi_{h}$ is the angle through the plasma with a laser hole. The solid lines in Fig. 1 show the densities at $v_{r}=0.1,0.04$, and 0.012 . The refraction method is sensitive to the hole diameter and has no strong dependence on the electron density at $<10^{22} \mathrm{~cm}^{-3}$. The refraction angle of the probe light is too large to use $20 \mathrm{~nm}$ as a probe wavelength in the plasma at densities of $>10^{22} \mathrm{~cm}^{-3}$. Figure 1 indicates the merit of the absorption method for a larger size vacuum hole in higher density plasmas. However, the laser-hole boring experiments will require the refraction method since the size of the laser hole with self-focusing will be smaller than the original focal spot diameter $(30-50 \mu \mathrm{m})$. The deflection method or refractometry using $20 \mathrm{~nm}$ light is more appropriate for the observation of several tens of $\mu \mathrm{m}$ hole at densities of $10^{20}-10^{22} \mathrm{~cm}^{-3}$ as compared with shadowgraphy.

\section{CONCEPT AND DESIGN OF THE XRL-GIR}

We applied the refraction technique using Ne-like Ge $\mathrm{x}$-ray laser light for the measurements of laser channeling because of the sensitivity of the technique to small density 


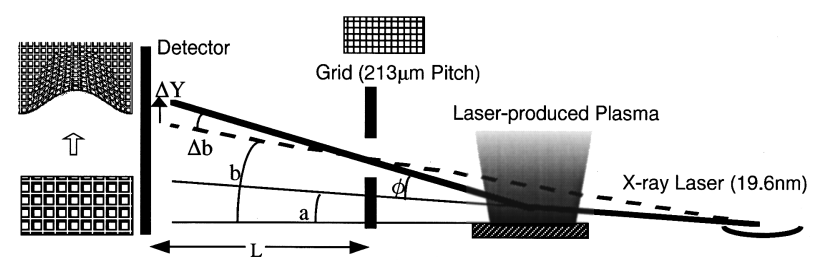

FIG. 2. Concept of the XRL-GIR technique to measure electron density profiles. The gradient of the refractive index in the probed plasma can be estimated from the grid distortion ( $\Delta b$ : angle, $\Delta Y$ : distance), resulting in evaluation of the density profiles. $\Phi$ : deflection angle; $a$ : original angle of the deflected beam to the target parallel; $b$ : no deflected beam angle parallel to the target.

perturbation as shown in Fig. 1. The GIR technique ${ }^{9}$ was used to obtain two-dimensional information on the density profiles. The Schlieren technique as one of the refraction methods also gives us a two-dimensional contrast image due to $\mathrm{x}$-ray deflection in the plasma. However, the requirement for probe-beam uniformity using GIR or moire is lower than that with the Schlieren method. A GIR method will be more appropriate to probe two-dimensional nonuniform plasmas than the moire method.

By using the GIR technique, two-dimensional distortion of the grid image is obtained as shown in Fig. 2. The grid distortion is caused by beam deflection in the gradient refractive index, which is sensitive to the electron density gradient. Defining $x$ as the axis along the plasma density gradient and $z$ as the orthogonal direction, the refraction angle $\phi$ is given by

$$
\phi=\int_{-\infty}^{Z_{0}} d z \frac{\partial N}{\partial x}
$$

where the integration is carried out along the $z$ path of the ray. $z_{0}$ is the total distance along the ray path and $N(x)$ the refractive index. If $N(x)$ has no dependence on bound electrons in an unmagnetized plasma, $N(x)$ is assumed to be

$$
N(x)=\sqrt{1-\frac{n_{e}(x)}{n_{c}}},
$$

where $n_{c}$ is the critical electron density corresponding to the probe-beam wavelength. The x-ray beam is deflected in the gradient plasma by an angle of $\phi$ from the straight line. A grid behind the plasma is illuminated by the beam deflected through the plasma and there is no deflection beam without any plasma. Comparing the distortion of the grid image and the no distortion grid image, we can estimate a distortion length $\Delta Y$ on the detector. ${ }^{10}$ From the shift $\Delta Y$, the refraction angle $\phi$ is obtained by

$$
\begin{aligned}
& \phi=(b-a)+\Delta b, \\
& L \Delta b=\Delta Y \cos (b) \cos (b-\Delta b) .
\end{aligned}
$$

Therefore, we can estimate two-dimensional electron density profiles by ray-trace calculations describing the distortion on the grid image obtained with the GIR.

This GIR technique was extended to XUV wavelengths by using a $19.6 \mathrm{~nm} \mathrm{Ne-like} \mathrm{germanium} \mathrm{x}$-ray laser developed at the Institute of Laser Engineering, Osaka University. ${ }^{11}$

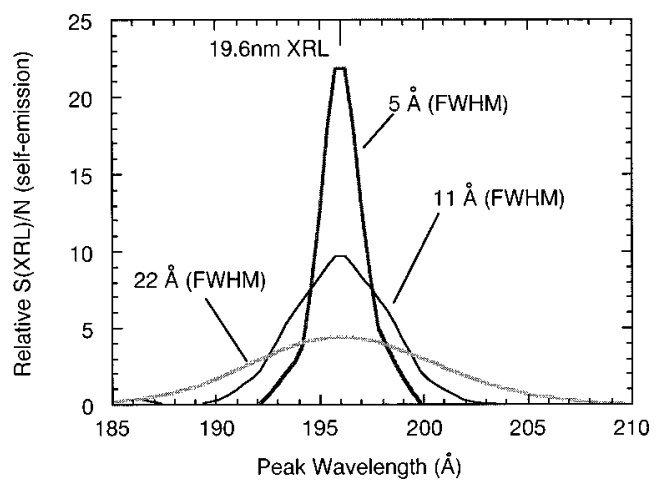

FIG. 3. Ratio of the x-ray laser signal intensity to the self-emission from $\mathrm{CH}$ plasmas changing the bandwidth and the center wavelength of the spectral response of the reflectivity of the $\mathrm{x}$-ray mirror.

The x-ray laser beam has a beam divergence of 3-4 mrad, a pulse duration of $80 \mathrm{ps}$, and a peak power of about $10 \mathrm{MW}$ at a wavelength of $19.6 \mathrm{~nm}$. We optimized the GIR system using the $19.6 \mathrm{~nm}$ laser light for measurements of laser channeling into overdense plasmas which was created on a plastic target $(\mathrm{CH})$. The $\mathrm{CH}$ plasma was created by laser light and the temperature was above $1 \mathrm{keV}$, resulting in no significant effect on the x-ray laser absorption through probing of the plasma at electron densities of less than $10^{22} \mathrm{~cm}^{-3}$. However, the signal intensity of the self-emission from the $\mathrm{CH}$ plasma might compete with the $\mathrm{x}$-ray laser signal even ignoring the $\mathrm{x}$-ray deflection. The spectral intensity of the $\mathrm{x}$ ray laser light from the Ge plasma is much higher than the intensity from the $\mathrm{CH}$ plasma while the spectral-integrated signal of the $\mathrm{CH}$ emission may be crucial for the measurement, which depends on the spectral band width of the collected $\mathrm{x}$-ray emission. In order to increase the signal to noise $(\mathrm{S} / \mathrm{N})$ ratio, we have used several pieces of multilayer $\mathrm{x}$-ray mirrors as filters. Figure 3 shows the relative value of the signal to noise ratio as a function of the peak wavelength and the width of the mirror reflectivity for $19.6 \mathrm{~nm}$ laser light taking into account the $\mathrm{x}$-ray spectra from the $\mathrm{CH}$ plasma. A narrower band width of mirror reflectivity is necessary to obtain a higher $\mathrm{S} / \mathrm{N}$ as well as matching of the center wavelength of the reflectivity to the $\mathrm{x}$-ray laser light. $\mathrm{Mo} / \mathrm{Si}$ multilayer mirrors were used to filter the $\mathrm{x}$-ray laser signal from the $\mathrm{CH}$ self-emission, which was also utilized for relaying the interaction plasma image. The peak reflectivity of the mirror was $35.5 \%$ at a wavelength of $19.65 \mathrm{~nm}$ with a bandwidth of $0.9 \mathrm{~nm}$ [full width at half maximum (FWHM)]. Four pieces of mirror were used to increase the S/N. A simple estimation indicates that the $\mathrm{x}$-ray laser signal would be 35 times as high as the spectral integrated intensity of the self-emission from the $\mathrm{CH}$ plasma through the mirrors. The estimation used the $\mathrm{x}$-ray spectral intensities of the $\mathrm{x}$-ray laser and the self-emission was obtained separately with a spectrometer. No refraction or absorption effect is taken into account in the estimation. Hence, the $\mathrm{S} / \mathrm{N}$ was about 3 in the experiments, indicating large refraction and/or absorption of laser light in the plasma. 


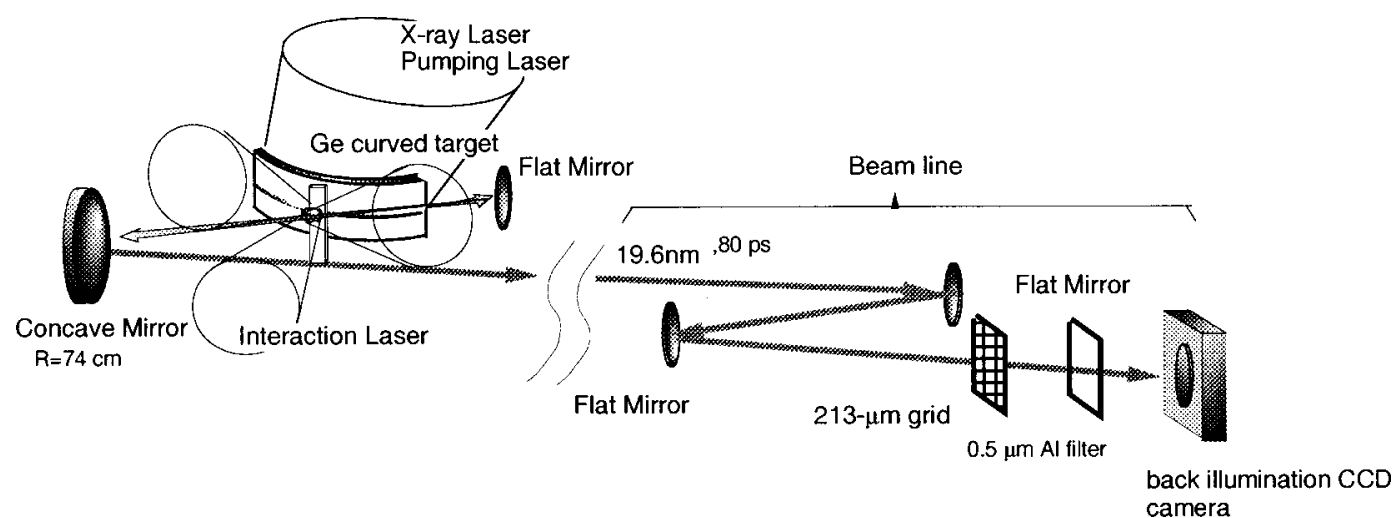

FIG. 4. Experimental setup for measurements of the electron density profiles of the laser channel into overdense plasmas using the XRL-GIR. The interaction $\mathrm{CH}$ plasmas were created behind the Ge curved target where the x-ray laser light was generated. Four pieces of x-ray mirror were used to select the x-ray laser signal and relay the image.

\section{EXPERIMENTAL SETUP}

The experiments were carried out with the Gekko XII laser system at Osaka University. A single beam of 1.053 $\mu \mathrm{m}$ laser light irradiated a Ge curved target at a $100 \mathrm{ps}$ double pulse with a $400 \mathrm{ps}$ time separation to generate 19.6 $\mathrm{nm}$ laser light into Ne-like Ge ions. The pumping energy for the $\mathrm{x}$-ray laser was $150-175 \mathrm{~J}$ per pulses. A single $\mathrm{X}$-ray laser pulse with a pulse duration of 80 ps (FWHM) was created at a time corresponding to the second pumping pulse. The x-ray laser beam was relayed onto an interaction target with a flat Mo/Si multilayer mirror and lit the target from the side as shown in Fig. 4. The x-ray image of the interaction plasma illuminated by the $\mathrm{x}$-ray laser light was relayed by a $74 \mathrm{~cm}$ diam spherical multilayer mirror onto an x-ray charge coupled device (CCD) camera through two flat multilayer mirrors and an aluminum filter with a thickness of $0.5 \mu \mathrm{m}$ in the beam line. The magnification of the imaging system was $12 \times$ from the interaction plasma to the imaging point. The collection limit of a deflected beam by the spherical mirror in the probe system was $7.5 \times 10^{-2} \mathrm{rad}$. The $\mathrm{x}$-ray image was detected with a back illumination type CCD camera (1100 $\times 330$ pixels with a pixel size of $24 \times 24 \mu \mathrm{m}$ ). The detectors were located $80 \mathrm{~cm}$ from the imaging point to increase the sensitivity of the deflection angle and to decrease the intensity of the self-emission as noise. A $213 \mu \mathrm{m}$ pitch mesh used as a grid was set $60 \mathrm{~cm}$ from the imaging point on the target side. The arrangement of the GIR probe system was optimized by a ray-trace calculation to diagnose a plasma with a scale length of $100 \mu \mathrm{m}$ at $10^{20}-10^{22} \mathrm{~cm}^{-3}$ electron density. A typical spatial resolution given by the grid size was evaluated to be about $10 \mu \mathrm{m}$.

Interaction plasmas were created on a $100 \mu \mathrm{m}$ thick $\mathrm{CH}$ target, which was precisely set at the back of the x-ray laser target as shown in Fig. 4. The interaction target was irradiated obliquely by two $0.351 \mu \mathrm{m}$ laser beams of a $100 \mathrm{ps}$ (FWHM) double pulse with an interval of $400 \mathrm{ps}$ to produce a preplasma at a $400 \mu \mathrm{m}$ focal spot $\left(10^{14} \mathrm{~W} / \mathrm{cm}^{2}\right)$. A 1.053 $\mu \mathrm{m}$ laser light used as a main interaction pulse was focused normally onto the preplasma after $1 \mathrm{~ns}$ of the first prepulse with an aspherical lens of $f / 3$. The intensity of the main pulse was $2 \times 10^{17} \mathrm{~W} / \mathrm{cm}^{2}$ with a $30 \mu \mathrm{m}$ spot at the best focusing position. The best focusing position was set 210 $\mu \mathrm{m}$ from the initial target surface. The x-ray laser light lit the plasma from the side $50 \mathrm{ps}$ after the peak of the first main pulse. The pulse shapes of the laser lights were monitored with biplanar photodiodes as being near Gaussian and having double pulse intensity ratios of $1: 1$. The formation of the channel was measured with an UV laser interferometer for underdense plasmas $\left(10^{19}-10^{20} \mathrm{~cm}^{-3}\right),{ }^{12}$ and the XRL-GIR system was used for the overdense plasmas. A $263 \mathrm{~nm}$ laser light with a pulse duration of $10 \mathrm{ps}$ was used for the UV interferometer. Properties of the laser turning point $\left(\sim 10^{21} \mathrm{~cm}^{-3}\right)$ were monitored by measurement of a Doppler shift of the backscattered light spectra with a streak camera coupled to a spectrometer. ${ }^{3}$

\section{EXPERIMENTAL RESULTS}

Figures 5(a) and 5(b) show, respectively, typical XRLGIR images of a $\mathrm{CH}$ target without any plasma and a $\mathrm{CH}$ plasma created by $0.35 \mu \mathrm{m}$ laser light at $10^{14} \mathrm{~W} / \mathrm{cm}^{2}$. The $\mathrm{x}$-ray laser beam probed the plasma $1 \mathrm{~ns}$ after the first pulse, corresponding to the time of the interaction of the channeling beam with the preformed plasma. Shadows of the target and grid images without any distortion are seen in Fig. 5(a) because there is no deflection by the x-ray laser beam. By probing the preformed plasma, the x-ray laser beam was refracted, resulting in distortion of the grid image as shown in Fig. 5(b). The preplasma with a $400 \mu \mathrm{m}$ diameter was created on the left-hand side of the target on the image. The $\mathrm{x}$-ray laser beam was deflected towards the direction of plasma expansion. Self-emission from the $\mathrm{CH}$ plasma was also detected behind the grid image as a weak background signal. No grid image detected near the target surface is ascribed to $\mathrm{x}$-ray deflection, with a large angle due to the steep density gradient and absorption in the high density plasma where the temperature is also low enough for the absorption.

Figure 6(a) shows a two-dimensional electron density contour of the $\mathrm{CH}$ plasmas from the XRL-GIR image. The two-dimensional profiles were evaluated by iteration of a ray-trace calculation to self-consistently fit it to the distorted grid image assuming no effects of a magnetic field and bound electrons on the refractive index. Another assumption 

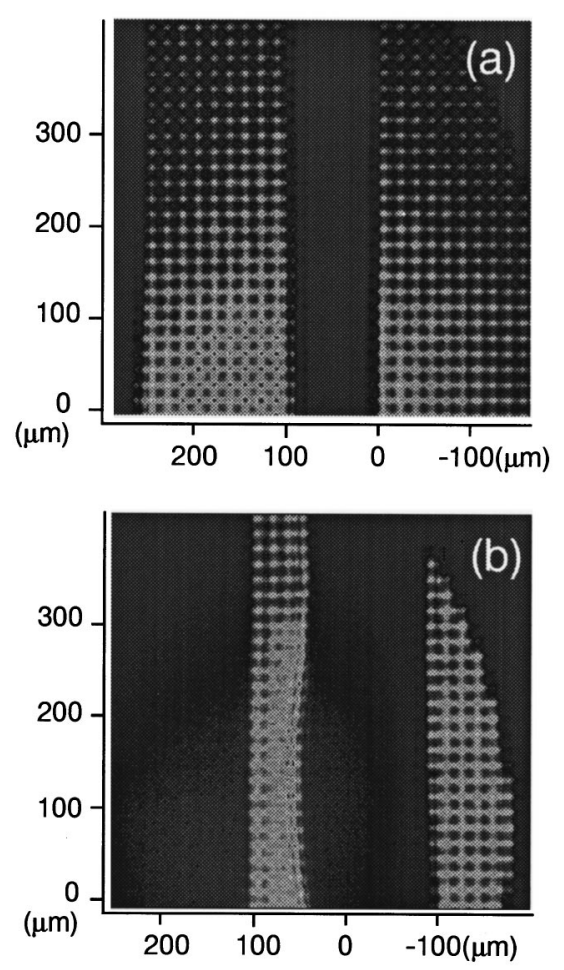

FIG. 5. Typical XRL-GIR images of (a) a $\mathrm{CH}$ target without any plasma and (b) a $\mathrm{CH}$ plasma created by $0.35 \mu \mathrm{m}$ laser light at $10^{14} \mathrm{~W} / \mathrm{cm}^{2}$ on the left-hand side of the target. The plasma was probed at $1 n s$ after the first pulse of the double pulse with the $\mathrm{x}$-ray laser light.

for the evaluation is a symmetrical structure of the preplasma setting the center normal axis in the focal spot as a rotational axis. The preformed plasma has a $\cos ^{0.5}$ distribution at near critical densities with a width of $400 \mu \mathrm{m}$ in diameter. Figure 6(b) shows the one-dimensional electron density profiles of the preplasma obtained with the XRL-GIR and with the UV interferometer. The one-dimensional profiles correspond to the densities along the plasma expansion at the center of the focal spot. The density profile from the XRL-GIR is consistent with the extrapolation from the profile obtained by the UV interferometer. The profiles have a double exponential shape with a scale length of $30-40 \mu \mathrm{m}$ at $10^{21} \mathrm{~cm}^{-3}$ and a scale length of about $300 \mu \mathrm{m}$ at $10^{20} \mathrm{~cm}^{-3}$. The solid lines in Fig. 6(b) indicate profiles from a one-dimensional hydrodynamic code (ILESTA) for different geometries [plane and spherical ( $R=2 R_{s}$, spot radius) geometry as an initial target] that take into account a three-dimensional effect on the plasma expansion. At higher density, the plane geometry is more consistent with the experimental result and the spherical geometry of $R=2 R_{s}$ gives us reasonable agreement of the profile with the experiment at lower density.

This XRL-GIR technique was used in laser-hole boring experiments. Figure 7(a) shows an XRL-GIR image of the interaction of the channeling beam $\left(2 \times 10^{17} \mathrm{~W} / \mathrm{cm}^{2}\right)$ with the preplasma as shown in Fig. 6(b). The channel beam was focused on the preplasma $210 \mu \mathrm{m}$ from the target surface. Clear grid distortions are seen in the image except for the distortion due to the plasma expansion. One kind of distortion appeared along the beam direction indicating ridges of density humps such as the plasma wall of the laser channel.
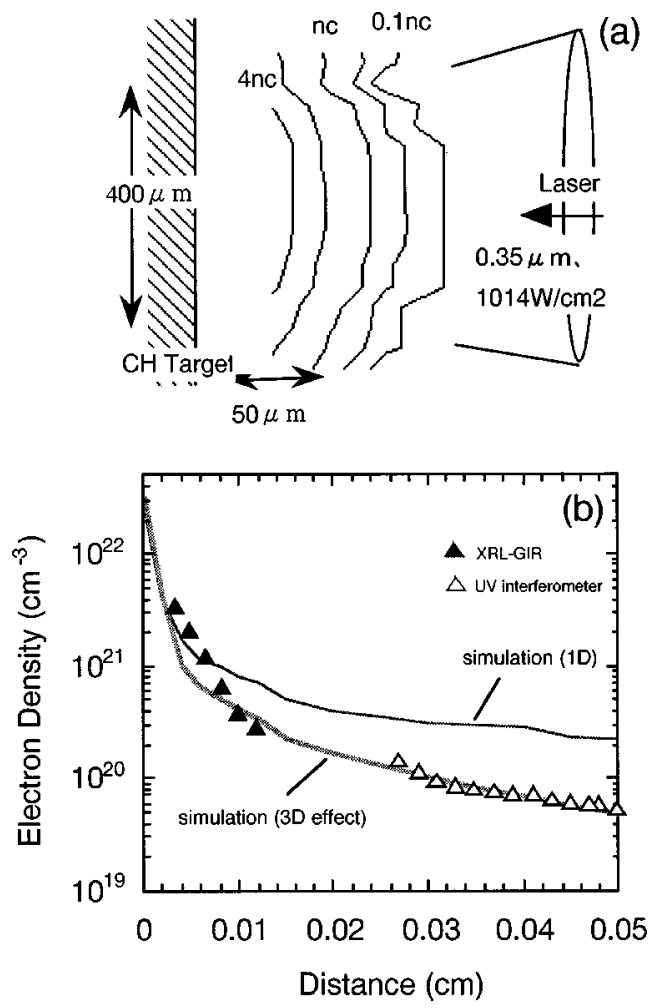

FIG. 6. (a) Two-dimensional electron density contour of the $\mathrm{CH}$ plasmas from the XRL-GIR image. (b) One-dimensional electron density profiles of the preplasma obtained with the XRL-GIR and with the UV interferometer. The solid lines indicate profiles of one-dimensional hydrodynamic simulation for different geometries [plane and spherical $\left(R=2 R_{s}\right)$ geometry].

Assuming the refractive index is given by only free electrons, density well and walls are obtained at overcritical regions on the electron density profiles from the grid images. The channel width was less than $30 \mu \mathrm{m}$ at 50-60 $\mu \mathrm{m}$ from the target surface. A width of the laser profile of less than 26 $\mu \mathrm{m}$ could be required to explain the density profile from a simple estimation given by an equilibrium condition between the the ponderomotive force and thermal pressure for 1-10 $\mathrm{keV}$. This result indicates laser self-focusing and channeling into overdense plasmas. Another ridge of density humps, diverging along the channel in the direction opposite to the laser beam, appeared at outer regions from the channel walls. Extrapolated lines of this ridge of the density hump cross at the target surface on the beam axis corresponding to the channel axis. These density humps may indicate a Mach cone of the shock waves created by supersonic propagation of the channel front. From a Doppler shift of the backscattered light spectra showing a front speed of $7 \times 10^{7} \mathrm{~cm} / \mathrm{s}^{3}$ the angle of the Mach cone is estimated to be $43^{\circ}$ for a plasma temperature of $3 \mathrm{keV}$. This Mach cone angle from the front speed is consistent with the experimental observation $\left(45^{\circ}\right)$. Details of the channel formation appear elsewhere. ${ }^{13}$ The other interesting distortion is a ball structure $150 \mu \mathrm{m}$ from the target surface. The grid pattern of this ball structure is radially distorted towards the outside. Assuming that the refractive index is given by free electrons only and that there is no effect of magnetic fields, the electron density is evaluated to be $6 \pm 4 \times 10^{21} \mathrm{~cm}^{-3}$ at the center 

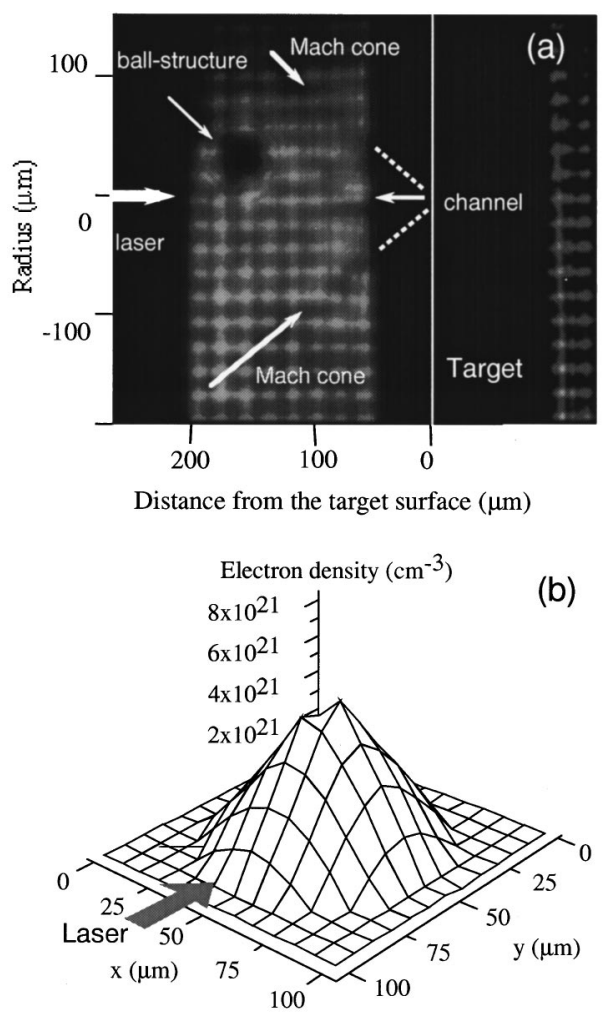

FIG. 7. (a) XRL-GIR image of the interaction of the channeling beam with the preplasma. (b) Electron density profile corresponding to the ball structure on the grid image assuming that the refractive index is given by only free electrons and that there is no effect of magnetic fields.

of the ball structure as shown in Fig. 7(b). As another possibility, this ball structure might be ascribed to the refractive index affected by a localized strong magnetic field due to hot electrons induced by laser self-focusing. The refractive index including the effect of the magnetic field is given by

$$
N=\left(1-\left(\omega_{p} / \omega\right)^{2} \frac{\omega^{2}-\omega_{p}^{2}}{\omega^{2}-\omega_{p}^{2}-\omega_{c}^{2}}\right)^{1 / 2}
$$

for the magnetic filed direction perpendicular to the ray direction of the probe beam. Another formula for the field direction parallel to the ray direction is given by

$$
N=\left(1-\left(\omega_{p} / \omega\right)^{2} \frac{1}{1-\left(\omega_{c} / \omega\right)}\right)^{1 / 2}
$$

where $\omega_{p}$ is the plasma frequency, $\omega$ the laser frequency, and $\omega_{0}$ the cyclotron frequency dependent on a magnetic field. A magnetic field of a few $\mathrm{G}$ (gauss) would be required to distort the $19.6 \mathrm{~nm} \mathrm{x}$-ray grid image into a ball structure as shown in Fig. 7(a) taking into account the gradient of the magnetic field in the refractive index. It will be difficult to create such a high magnetic field even though laser intensity is enhanced by a factor of 10 in self-focusing and channeling. The x-ray image of self-emission from the point corresponding to this ball structure was also obtained with an $\mathrm{x}$-ray pinhole camera. More details of the ball structure should be studied in the future.

\section{SUMMARY}

We developed an XUV (19.6 nm) laser grid-image refractometer (XRL-GIR) to diagnose laser-hole boring into overdense regions. The optical layout of the XRL-GIR was optimized to measure the $100 \mu \mathrm{m}$ scale-length plasma at electron densities of $10^{20}-10^{22} \mathrm{~cm}^{3}$. The density profiles obtained by the XRL-GIR were compared with those at $10^{19}-10^{20} \mathrm{~cm}^{3}$ from an UV interferometer, resulting in good continuity of the profiles from one to the other. By using this XRL-GIR, we directly observed laser channeling into overdense plasmas for the first time. The two-dimensional profiles show laser self-focusing in the overdense region and density evacuation in the channeling by ponderomotive forces. We also obtained a Mach cone created by supersonic propagation of the channel front. Shorter pulse durations of the $\mathrm{x}$-ray laser light such as a few ps will be required to obtain a high contrast image of the channel with high temporal resolution since the channel front propagates into overdense regions at a speed of $7 \times 10^{7} \mathrm{~cm} / \mathrm{s}$.

\section{ACKNOWLEDGMENTS}

This work was performed under the technical support of the glass laser operation group, the target fabrication group, and the data acquisition group at the Institute of Laser Engineering (ILE), Osaka University. The authors wish to acknowledge technical support by Dr. M. Takagi and they thank Dr. A. S. Wan at Lawrence Livermore National Laboratory and Dr. H. Daido at ILE for useful comments. One of the authors (K.T.) was supported by research fellowships from the Japan Society for the Promotion of Science.

${ }^{1}$ D. Attwood et al., Phys. Rev. Lett. 40, 184 (1978); A. Raven et al., ibid. 43, 278 (1979).

${ }^{2}$ P. E. Young, Phys. Fluids B 3, 2331 (1991); S. Wilks et al., Phys. Rev. Lett. 73, 2994 (1994); P. E. Young et al., ibid. 75, 1082 (1995); M. Borghesi et al., ibid. 78, 879 (1997); J. Fuchs, et al., ibid. 80, 1658 (1998).

${ }^{3}$ R. Kodama et al., Phys. Rev. Lett. 77, 4906 (1996); K. Takahashi et al., Opt. Commun. 135, 45 (1996).

${ }^{4}$ D. L. Matthews et al., Phys. Rev. Lett. 54, 110 (1985); A. Carillon et al., ibid. 68, 2917 (1992); L. B. DaSilva et al., Opt. Lett. 18, 1174 (1993).

${ }^{5}$ D. Ress et al., Rev. Sci. Instrum. 66, 579 (1995).

${ }^{6}$ M. Key et al., J. Quant. Spectrosc. Radiat. Transf. 54, 221 (1995).

${ }^{7}$ L. B. DaSilva et al., Phys. Rev. Lett. 74, 3991 (1995); A. S. Wan et al., Phys. Rev. E 55, 6293 (1997).

${ }^{8}$ Y. B. Zel'dovich and Y. P. Raizer, Physics of Shockwaves and High Temperature Hydrodynamic Phenomena (Academic, New York, 1966).

${ }^{9}$ R. S. Craxton et al., Phys. Fluids B 5, 4419 (1993).

${ }^{10}$ K. Takahashi et al., Proc. SPIE 3156, 146 (1997).

${ }^{11}$ R. Kodama et al., Phys. Rev. Lett. 73, 3215 (1994).

${ }^{12} \mathrm{~K}$. Mima et al., Phys. Scr. (to be published).

${ }^{13} \mathrm{~K}$. Takahashi et al., ILE Research Report No. ILE9811P, 1998. 